\title{
Joint Deblurring and Denoising of Hyperspectral Images with PCA and Totalvariation
}

\author{
K.E.Sreenivasa Murthy, R.Sudheer Babu, Shaik Saheb Basha
}

\begin{abstract}
Human imaginative and prescient is an excellent imaging shape that can capture and disentangle mild imperativeness beginning from one-of-a-kind assets regardless of the way that it's miles constrained to observable mild. There arevarious programs, as an instance, face acknowledgment, restorative imaging, agribusiness, geology,surveillance, and so on that advantages via imaging a few companies of the electromagnetic spectrumoutside the noticeable range. The hyperspectral imaging strategies are able ofcapturing many agencies of the electromagnetic range and in this way, can be consideredas the speculation of shading imaging. on this paper, we commonly have a tendency to spark off a totally explicit plan for hyper-ghastly (HS) photo deblurring with overwhelming attitude appraisal (PCA) and everyday variety (tv). we have a penchant to introductory decorrelate the HS images and separate the insights content material material from the clamor with the manual of utilizing manner that of PCA. At that factor, we generally will in popular observe the tv approach to conjointly denoise and deblur the principle transcendent components (pc frameworks). After, commotion within the ultimate primary additives is smothered the utilization of a clean delicate thresholding point, for device execution. initial outcomes on reproduced and authentic $\mathrm{HS}$ photos location unit specifically encouraging.
\end{abstract}

Catchphrases :multi-define picture excellent-dreams, denoising, electromagnetic range, complete variety.

\section{PRESENTATION}

Hyperspectral imaging accumulates facts from over the variety. the objective of hyperspectral imaging is to inspire variety of each constituent of the scene which can be then wont to observe or watch objects, installation things and know-how methods that region unit happening inside the vicinity of intrigue [1][2]. The human tangible framework recognizes the shade of daylight in an a large portion of 3 companies, excessive wavelengths - handled as red, medium wavelengths - handled as unpracticed and lower wavelengths - treated as blue. anyway the otherworldly scanners elements it into some companies. this association of tearing into some groups will just reached out to the exchange this is imperceptible. The hyperspectral photos have a awesome undulating goals and consists of a greater substantial

Differ of wavelengths. It incorporates both adjacent agencies and divided corporations [3].

Dr. K.E.Sreenivasa Murthy, Professor and Head, ECE Department, G.Pullaiah College of Engineering and Technology, Kurnool-518452, AP, India.

Dr. R.Sudheer Babu, Assistant Professor, ECE Department, G.PullaReddy Engineering College, Kurnool-518007, AP, India.

Dr. Shaik Saheb Basha, Professor,ECE Department, G.PullaReddy Engineering College, Kurnool-518007, AP, India.
Revised Manuscript Received on July 18, 2019.

Makes use of of hyperspectral imaging consists of some fields. In agribusiness, the harvest reputation, expire id, tempo of yield, spreading fee of any perish is probably exhausted a gifted strategies using hyperspectral images. In medicinal field, in particular eye care is in particular executed exploitation hyperspectral pix. additionally to the consideration care, malignant boom, tumors, inadequacy of different organs of the human in like manner as creature body is probably done successfully exploitation hyperspectral images. Sustenance process is another area any region the hyperspectral images territory unit being used. antiquated cameras and optical machine sorters forget about to apprehend a few imperceptible deformity and far flung substances. Hyperspectral pix region unit applied right here to remove deformities and outdoor substances subsequently raising the same old of sustenance and product. topography makes use of hyperspectral photos to recognize a few minerals from transportable photos. the arrangement and available degree of minerals additionally can be precisely measurable by procedure the hyperspectral photos. police exam, cloth technology, Astronomy, Chemical imaging and putting region unit the contrary large zones any place the hyperspectral pictures see applications. by way of and massive those instances, the commotion that adulterates the main clean photograph, activates poor estimation of any article in the photograph. as a result denoising of hyperspectral image is of decent test. In hyperspectral picture denoising, furthermore to the spacial statistics extraordinarily associated otherworldly statistics should also be contemplated.

The got brilliance at the an extended way off detecting hyperspectral automatic digitaldigital digicam is debased via way of the usage of environmental consequences and instrumental commotions. The barometrical impacts need to be remunerated to provide the reflectance. Instrumental (sensor) commotion carries heat (Johnson) clamor, quantization clamor and shot (photon) clamor which intent debasement in the ghostly companies via the utilization of utilising the usage of various extents. the ones undermined businesses debase the efficiency of the HSI assessment methodologies and as a result they may be commonly disbursed with from the insights beforehand of time than any what is more making ready. as an alternative, HSI denoising is probably considered as a preprocessing assignment in HSI evaluation to upgrade the sign to commotion percentage (SNR) of HIS

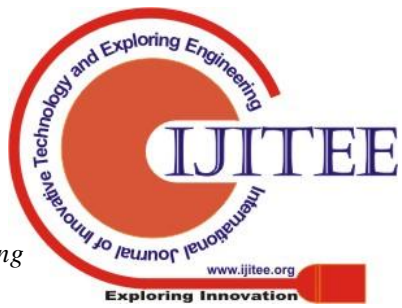




\section{JOINT DEBLURRING AND DENOISING OF HYPERSPECTRAL IMAGES WITH PCA AND \\ TOTALVARIATION}

In this fragment, we have have been given had been given investigated one-of-a-kind patterns for motivation denoising uniquely union in advance of time and appraisal in advance of time and tentatively determined that amalgamation going before rendition yields higher effects than assessment past variation. the second one dataset altered into stuck over a rustic place within the south of the city of Trento, Italy. This dataset is of length 600 with the beneficial asset of the utilization of utilizing 166 pixels with a spatial selection of aftermarket with sixty three ghastly groups (starting from 402.89 to $989.09 \mathrm{~nm}$ ) stuck thru the AISA Eagle sensor. The available groundtruth covers six instructions of intrigue all in all with building, Woods, Apple wood, Roads, winery, and floor. determine 4 represents a phony shading composite occasion of the hyperspectral insights and the referring to making ready and test checks. table 3provides measurements across the relating fashion of training and check out exams. we have a tendency to take out the clamor (with out deblurring) within the stop PCA channels the use of a smooth-thresholding trouble. The results show viability of the intentional methodology every outwardly and quantitatively.

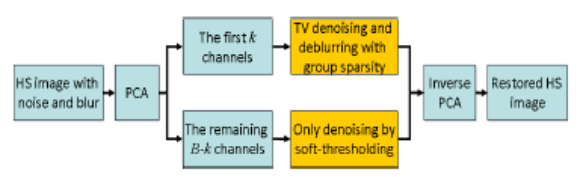

Fig. 1: Flow chart of the proposed deblurring method

\section{PROPOSED APPROACH \& RESULTS}

HSI denoising is a sensitive undertaking and desires specific interest in evaluation to denoising of various snap shots because of the importance of maintaining up ghostly records. the unconventional ghastly courting gives an exceptionally first rate growth for the denoising mission, in any case, over smoothing idea methods absence of sizable ghostly facts. in the ensuing segments, we viewpoint out the statute problems related with the improvement of HSI

Denoising calculations. $\mathrm{H}$ the straight haze administrator. The maximum intense specific reply answer, for HS picture deblurring, is to apply the following appropriately advanced development inconvenience to reestablish every one-of-akind phantom band in my view:

$$
\widehat{u}_{i}=\operatorname{argmin}_{u_{i}}\left|\nabla u_{i}\right|+\frac{\beta}{2}\left\|H u_{i}-f_{i}\right\|_{2}^{2}
$$

The selection of the HSI model requires intrigue. for instance, in version (2), painstakingly decided on for a modern normal run of the mill by way of and large execution of HSI denoising. further, the choice of the version parameters is certifiably now not an inconsequential errand. for example, finished or under assessing records misfortune or horrendous denoising normal execution. offering ad hoc or exploratory strategies for the ones estimations can likewise besides moreover make denoising calculations temperamental and convoluted to be used as a preprocessing assignment in HSI assessment. it is pleasantly extraordinarily well worth refering to that choosing step by step complicated fashions or impacts settles at the parameter selection project hundreds harde parameter $\beta \_$icontrols the

overall commitment of the certainties stability timespan and smoothing time frame.

Ghastly insights in HSIs is of dynamite centrality in HSI evaluation. as a result, it is basic that HSI denoising techniques preserve up the otherworldly information. every signal and clamor fluctuations are diverse at some reputation inside the hyperspectral companies, which makes the commotion estimation and denoising task difficult. to conform to this problem, some denoising strategies use band-astute standardization to earnings ghastly corporations of comparative scale. Band-affordable standardization concept methods unearthly twisting and it is not embraced for HSI. One method to cope with numerous signal and commotion modifications is to define the form parameters to be variable w.r.t. the ghastly businesses.

$$
\hat{u}_{i}=\operatorname{argmin}_{u_{i}} \sum_{j=1}^{B}\left\|\Delta u_{i}\right\|_{2}+\frac{\beta}{2}\left\|H u_{i}-f_{i}\right\|_{2}^{2}(2)
$$

Denoising strategies, and uniquely the version parameter inclination necessities, are always principally strong on the estimation of the commotion distinction. one of the most famous methods utilized for HSI clamor parameter estimation is a couple of straight relapse (MLR) [11,12]. MLR changed into proposed in as a clamor estimation technique which assume that every band is a right away combination of the open door groups and in this way is probably expected with the treasured beneficial manual of the utilization of least squares estimation. the critical cause of the accomplishment of MLR is the unconventional ghastly connection of the pixels. This technique does no longer revel in in musings spatial data. be that as it is able to, traditional commotion fluctuation estimation systems, which consist of the center estimator completed at the wavelet coefficients [14] exceptional bear in mind the spatial connections. consequently, it's miles important to take a look at distinction clamor estimation procedures which make the finest each phantom and spatial relationships. $\hat{u}_{i}^{P C}=\operatorname{argmin}_{u_{i} P C}\left|\nabla u_{i}^{P C}\right|+\frac{\beta_{i}}{2}|| H u^{P C}{ }_{i}-$

fPCi2Zbesides the giant development in HSI recuperation structures, they have got typically now not been utilized in HSI evaluation as a preprocessing step. this will be due to numerous reasons which incorporates the computational fee, efficiency, unwavering best, and mechanization of the calculations. the primary motive of the denoising-based totally completely preprocessing degree is to decorate the SNR of the determined dataset. it is crucial to analyze the dedication of the various HSI recovery methods as a preprocessing challenge for likewise HSI appraisal, along exchange discovery, choice improve, classification or unmixing. application.

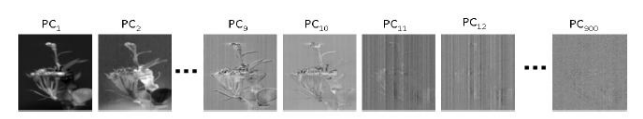

Fig. 2: Principal components of real HS image 
Inside the route of the beyond couple of years, a big amount of research has been dedicated to hyperspectral picture denoising. conventional denoising strategies based totally on second modeling and convex optimization techniques were not efficient for HSI due to the truth the ones forget approximately aboutabout the spectral data. The drastically correlated spectral bands in HSI had been determined very useful to improve HSI denoising. As a save you surrender give up end result, HSI denoising techniques have evolved to techniques that include spectral information. the ones HSI denoising strategies may be labeled in 4 most vital businesses, so one can be treated underneath.

$\hat{u}_{i}^{P C}=\operatorname{argmin}_{u_{i} P C} \sum_{j=1}^{k}\left|\nabla u_{i}^{P C}\right|_{2}+\frac{\lambda_{1}}{2}\left\|H u^{P C}{ }_{i}-f^{P C}{ }_{i}\right\|_{2}^{2}(4)$

and denoising is only conducted on the remaining $B-k$ $\mathrm{PCs}$ (we call this method PCA $\mathrm{A}_{\mathrm{TVTV}}$ in the following) through thefollowing estimation:

$\hat{u}_{i}^{P C}=\operatorname{argmin}_{u_{i} P C} \sum_{j=k+1}^{B}\left|\nabla u_{i}^{P C}\right|_{2}+\frac{\lambda_{2}}{2}\left\|u^{P C}{ }_{i}-f^{P C}{ }_{i}\right\|_{2}^{2}(5)$

3-D model-primarily based without a doubt completely HSI denoising strategies utilize version (2), in which every the spatial and ghastly projections the usage of frameworks An and M , in my opinion are connected. The projection lattices An and $\mathrm{M}$ are usually decided straightforwardly to decorrelate the sign spatially and frightfully, one at a time, and for this every word reference or bases are applied. HSI recovery approaches dependent on three-D filtering are named in this enterprise. 3. Trial influences $\mathrm{A}$ in particular tainted band from the recreated dataset turn out to be determined on for an obvious appraisal of the rebuilding strategies in determine three. 2d-Wavelet demonstrates an genuinely horrendous preferred ordinary by means of and huge execution, which is not surprising, for the reason that denoising is completed on each phantom band for my part and the statistics is in particular ruined in that specific band. 3-D-Wavelet eminently improves the major remarkable, due to the fuse of the measurements from specific groups via three-d demonstrating and filtering. FORPDN, NAILRMA and HyRes all whole super. The slanted conventional ordinary common normal execution of LRMR on this band is a direct end result of reality that it is not meant to adapt to the form of the commotion alternate at multiple diploma in the ghastly groups. We except completed the denoising processes on a true dataset. discern 3 demonstrates the substantive assessment of the formerly mentioned hyperspectral denoising methods finished on the Trento dataset. a few portion of Band 59 is picked for the assessment resulting from the truth it is deliberately debased through manner of commotion. The results propose a almost same behavior as at the reproduced dataset. 2nd Wavelet plays out the weakest, on the equal time as FORPDN, NAILRMA and HyRes accumulate the astonishing sizeable exhibitions. centered inside the underlying fine laptop frameworks. on this degree, we've got a have an investigate the impact of the HSI denoising structures as a preprocessing task for HSI classification. at the off chance that you want a have a examine the general everyday general execution of severa denoising techniques, we've had been given related 3 acclaimed classifiers which joins asset vector machines (SVM) arbitrary lush place (RF) classifiers , and extreme contemplating machines (ELM) .SVM tries to split tutoring tests having a place with amazing guidelines through finding maximum edge hyperplanes within the multidimensional capability district wherein the examples are mapped SVM have been on the start conveyed to cure instantly classification inconveniences. be that as it can, they may be summed as much as nonlinear want skills via contemplating the so-referred to as component stunt. A element primarily based actually definitely actually SVM (making use of the Radial premise work (RBF) portion) extends the pixel vectors legitimate appropriate immediately right into a better dimensional spot in which the accessible examples are straightly detachable and gauges greatest facet hyperplanes in this new area inside the event which you want to upgrade the direct detachability of statistics.is an outfit method (a tough and brief of tree-like classifiers) in light of on want wood for classification. group classifiers run numerous (a gathering of) classifiers which may be as a long way because it subjects for me educated, after which the character outcomes are combined through a voting technique. preferably, a RF classifier must be an unprejudiced and indistinguishably precise randomization of slanted unpracticed mother and father. The RF classifies an input vector with the helpful manual of via taking walks down every longing tree (an excessive and quick of paired choices) within the lush zone (the arrangement of all timber). each tree consequences in a unit vote in want of a particular tastefulness and the lush vicinity alternatives this.
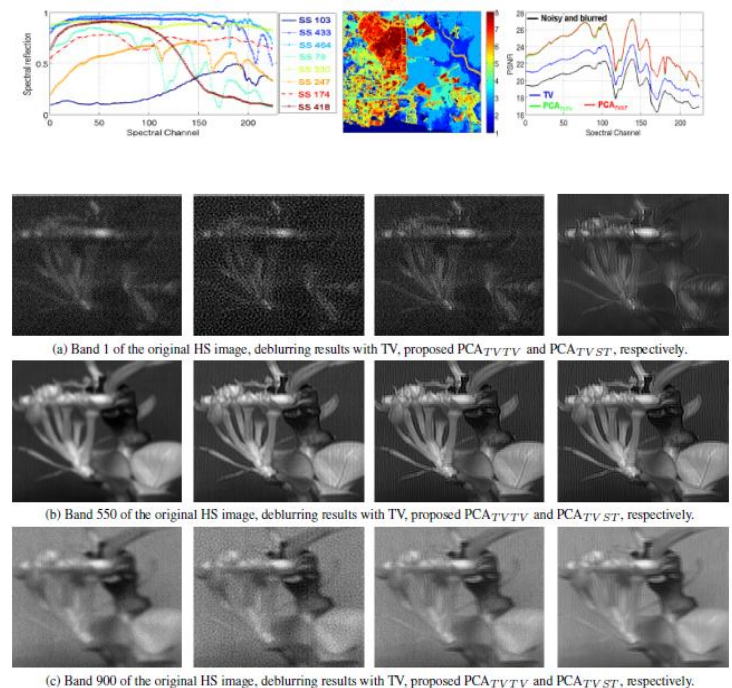

Fig. 4: Deblurring result on the real hyperspectral data

\section{CONCLUSION}

A totally certainly one of a kind deblurring approach for hyperspectral images is proposed in this paper. We beginning make use of a PCA upgrade to decorrelate the HS photo and to component the data content material cloth from 


\section{JOINT DEBLURRING AND DENOISING OF HYPERSPECTRAL IMAGES WITH PCA AND \\ TOTALVARIATION}

the commotion. we've a penchant to then utilize a short television approach with bunch meager condition to denoise and deblur earnestly the primary o.k. pcs, and a gentle thresholding denoising issue to in reality eliminate clamor inside the closing B - okay computers. Exploratory results on recreated and proper HS photographs reveal the presentation of the proposed technique.

\section{REFERENCES}

1. Yusuke Monno, Masayuki Tanaka, and Masatoshi Okutomi. Multispectral Demosaicking the use of Adaptive Kernel Upsampling. In IEEE Int. Conf. image manner., pages 31573160, 2011. ISBN 9781457713033.

2. Yusuke Monno, Masayuki Tanaka, and Masatoshi Okutomi. Multispectral Demosaicking using Guided filter. In IS\&T/SPIE Electron. Imaging, extent 8299, pages 82990o_- 82990o, jan 2012.

3. FumihitoYasuma, TomooMitsunaga, Daisuke Iso, and Shree ok Nayar. Summed up diverse Pixel camera: Postcapture control of resolution, Dynamic range, and Spectrum. IEEE Trans. image technique., 19(9):2241-53, sep 2010. ISSN 1941-0042. doi: 10.1109/TIP.2010.2046811.

4. Miroslav Kubat, Robert C Holte, and Stan Matwin. AI for the place of oil slicks in satellite tv for pc radar photos. Mach. research., 30(2-3):195-215, 1998.

5. Ira Leifer,William J Lehr, Debra Simecek-Beatty, Eliza Bradley, Roger Clark, and Others. high-quality in magnificence satellite tv for $\mathrm{pc}$ and airborne marine oil slick faraway detecting: application to the BP Deepwater Horizon oil slick. remote Sens. Environ., 124:185-209, 2012.

6. SajadFarokhi, Usman Ullah Sheik, Jan Flusser, and Bo Yang. close infrared face acknowledgment using Zernike minutes and Hermite portions. Inf. Sci., 316:234- - 245, 2015.

7. Jiayi Ma, Ji Zhao, Yong Ma, and Jinwen Tian. Nonunbending unmistakable and infrared face enlistment through regularized Gaussian fields popular. example Recognit., 48(3):772-784, 2015.

8. Haitao Zhao and Shaoyuan sun. Scanty tensor placing based multispectral face acknowledgment. instance Recognit., 133:427-436, 2014.

9. Juliane Bendig, Kang Yu, Helge Aasen, Andreas Bolten, Simon Bennertz, JaninBroscheit,Martin L Gnyp, and Georg Bareth. CombiningUAV-based totally plant variety from harvest floor fashions, apparent, and near infrared flowers records for biomass staring at in grain. Int. J. Appl. Earth Obs. Geoinf., 39:79-87, 2015.

10. Bingfang Wu, Jihua Meng, Qiangzi Li, Nana Yan, Xin Du, and Miao Zhang. faraway detecting primarily based international harvest checking: encounters with China's CropWatch framework. Int. J. Digit. Earth, 7(2):113 - - 137, 2014. 